\title{
Impact of Co-Site Interference on L/C-Band Spectrum for UAS Control and Non-Payload Communications
}

\author{
Robert J. Kerczewski \\ NASA Glenn Research Center \\ 21000 Brookpark Rd., MS 54-1 \\ Cleveland, OH 44135 \\ $+12164333434$ \\ rkerczewski@nasa.gov
}

\author{
William D. Bishop \\ Jacobs Engineering, FNS 21000 \\ Brookpark Rd., MS 54-1 \\ Cleveland, OH 44135 \\ +12164333473 \\ william.d.bishop@nasa.gov
}

\author{
Douglas J. Hoder \\ NASA Glenn Research Center \\ 21000 Brookpark Rd., MS 7-1 \\ Cleveland, OH 44135 \\ +12164333438 \\ douglas.j.hoder@nasa.gov
}

\author{
Kurt A. Shalkhauser \\ NASA Glenn Research Center \\ 21000 Brookpark Rd., MS 54-4 \\ Cleveland, OH 44135 \\ +12164333452 \\ kurt.a.shalkhauser@nasa.gov
}

\author{
Jeffrey D. Wilson \\ NASA Glenn Research Center \\ 21000 Brookpark Rd., MS 54-1 \\ Cleveland, OH 44135 \\ +12164333513 \\ jeffrey.d.wilson@nasa.gov
}

\begin{abstract}
In order to provide for the safe integration of unmanned aircraft systems into the National Airspace System, the control and non-payload communications (CNPC) link connecting the ground-based pilot with the unmanned aircraft must be highly reliable. A specific requirement is that it must operate using aviation safety radiofrequency spectrum. The 2012 World Radiocommunication Conference (WRC-12) provided a potentially suitable allocation for LOS CNPC spectrum in C-Band at 5030-5091 $\mathrm{MHz}$ band which, when combined with a previous allocation in L-Band (960-1164 MHz) may satisfy the LOS spectrum requirement and provide for high reliability through dual-band redundancy. However, the LBand spectrum hosts a number of aeronautical navigation systems which require high-power transmitters on-board the aircraft. These high-power transmitters co-located with sensitive CNPC receivers operating in the same frequency band have the potential to create co-site interference, reducing the performance of the $\mathrm{CNPC}$ receivers and ultimately reducing the usability of the L-Band for CNPC. This paper examines the potential for co-site interference, as highlighted in recent flight tests, and discusses the impact on the UAS CNPC spectrum availability and requirements for further testing and analysis.
\end{abstract}

\section{TABLE OF CONTENTS}

1. INTRODUCTION .1

\section{L-BAND OCCUPANTS AND CO-SITE} INTERFERENCE POTENTIAL

3. CNPC TEST RESULTS .2

4. IMPACT ON CNPC SPECTRUM................9

5. NEXT STEPS..................................................10

6. SUMMARY ..................................................11

REFERENCES.......................................................11

BIOGRAPHIES................................................12

\section{INTRODUCTION}

The goal of enabling the safe integration of unmanned aircraft systems (UAS) into the National Airspace System (NAS) is being approached with some urgency by the UAS industry and the Federal Aviation Administration (FAA), with research organizations such as NASA contributing significant research, development and testing activities. At NASA's Glenn Research Center, the focus is on the control and non-payload communications (CNPC) link which connects the pilot on the ground with the unmanned aircraft (UA) in flight, providing command and control signals to the aircraft and relaying aircraft telemetry to the ground.

A specific requirement for the CNPC link is that it must operate using aviation safety radiofrequency spectrum. Two types of links are required - line-of-sight (LOS) using terrestrial-based communications and beyond-line-of-sight (BLOS) using satellite communications. The 2012 World Radiocommunication Conference (WRC-12) provided an allocation for LOS CNPC spectrum in C-Band (5030-5091 $\mathrm{MHz}$ ) which, when combined with a previously existing allocation in L-Band (960-1164 MHz) may satisfy the LOS spectrum requirement. The particular portion of L-Band proposed for CNPC use is $960-977 \mathrm{MHz}$. This portion is only occupied by ship-board navigation systems and is essentially unused over land. The 5030-5091 MHz band is also allocated for BLOS CNPC, but since a significant portion of that band is required for LOS CNPC, additional BLOS spectrum would be required. More critically, there are no satellites in operation or in development to provide such services in that 
band. Hence BLOS CNPC cannot be provided in protected aviation spectrum under current conditions.

The situation is further complicated by uncertainty in the ability to fully use the L-Band LOS CNPC spectrum due to co-site interference. Co-site interference can occur when different communications, navigation or surveillance systems located on the same aircraft that are not sufficiently separated in frequency and physical location cause interference with each other. For example, a strong transmitter (supporting a communications link, radar system, automatic dependent surveillance-broadcast (ADS-B) system, etc.) operating in a nearby frequency band may produce out-of-band emissions sufficiently strong enough to interfere with sensitive receive equipment on the same aircraft. For UA, which may be relatively small, it may not be possible to physically isolate the two systems sufficiently in order to avoid co-site interference.

For UAS, the coexistence of several navigation and surveillance aviation systems in L-Band presents a potentially serious co-site interference issue. In particular, two different ADS-B systems may be required on UA for integration into the NAS: Mode S Extended Squitter, operating at $1090 \mathrm{MHz}$ and Universal Access Transceiver (UAT), operating at $978 \mathrm{MHz}$. In general, Mode S is intended for aircraft that operate at high altitudes (above 18000 feet) while UAT is intended for aircraft that operate at lower altitudes. In both cases, ADS-B transmitters have the potential to create co-site interference into UAS CNPC receivers operating in the 960-977 $\mathrm{MHz}$ portion of L-Band, with the UAT system at $978 \mathrm{MHz}$ being potentially the most problematic.

Flight tests performed by NASA Glenn Research Center with a prototype CNPC system in L-Band have confirmed some potential co-site interference. The source of the interference is still being investigated, but it has occurred subsequent to the installation of new ADS-B equipment on the NASA UAS test aircraft, although other flight test differences render it unclear as to whether interference existed on previous flight tests. The co-site interference problem is currently being studied with prototype UAS equipment in both laboratory and flight test settings. Further analysis is underway based on system specifications, standards and simulations.

This paper will provide background information on the Lband co-site environment; results of the testing to date; consideration of the impact of co-site interference on the UAS CNPC spectrum; and requirements for further testing and analysis.

\section{L-BAND OCCUPANTS AND CO-SITE INTERFERENCE POTENTIAL}

The use of L-Band for new aeronautical communications systems has necessarily required the analysis of co-existence issues between proposed new systems and existing systems.
Several excellent analyses of system characteristics and interference issues are provided in [1, 2, 3].

RTCA Special Committee 203 has considered co-site interference issues specifically related to CNPC links [1]. Currently RTCA Special Committee 228 is continuing this work and is recommending testing and analyses be initiated to fully explore and understand the co-site interference problem in L-band as it relates to CNPC.

Eurocontrol has studied L-Band co-site interference issues in order to enable aeronautical communications links to be deployed in other portions of L-Band [2, 3]. Therefore it is not necessary to repeat this information. We provide instead an overview of the relevant L-Band navigation systems that may be needed for civil UA operations. Military systems such as JTIDS and TACAN are not currently part of this assessment as it is focused on civil applications.

\section{Airborne Services Definitions}

The following are brief descriptions of the L-Band navigation systems under study.

DME - Distance Measuring Equipment is a navigational aid which interrogates ground stations using pairs of RF pulses. The airborne transmitter operates in the $1025-1150 \mathrm{MHz}$ range. Each pulse is $0.5 \mathrm{MHz}$ wide centered on the channel frequency. Upon receiving the interrogation, the ground station waits 50 microseconds and then replies. The airborne DME receiver then calculates the round trip delay providing the user with distance information.

SSR - Secondary Surveillance Radar provides surveillance information replying to interrogation by ATC using blocks of data pulses. The airborne transmitter operates at $1090 \mathrm{MHz}$. There are three modes: A, C and S. Mode A and Mode C provide aircraft specific information, primarily identification and flight data upon interrogation by ATC. Mode S contains features of Mode A and C, but also have capabilities to reply with more discrete information, such as the aircraft's 24-bit address.

UAT - Universal Access Transceiver provides horizontal position and velocity (State Vector) and other information (ADS-B message) to the ground to be used to provide the appropriate aeronautical uplink information, such as weather. The UAT data link operates at $978 \mathrm{MHz}$ and supports a signaling rate of $1 \mathrm{Mbps}$. The airborne transmitter sends one ADS-B burst each second.

ADS-B - Automatic Dependent Surveillance - Broadcast automatically provides (broadcasts) State Vector, intended flight path, aircraft type and other information that is shared with other aircraft with ADS-B In capabilities. ADS-B can be used in all phases of flight and include airport surface operations. The carrier frequency is $1090+/-1 \mathrm{MHz}$ and information is transmitted as Mode S Extended Squitter messages. 
Table 1 - Airborne Transmitter Specifications for L-Band Civil Navigation Systems

\begin{tabular}{|c|c|c|c|c|c|}
\hline \multicolumn{9}{|c|}{ Airborne Transmitter Specifications } \\
\hline System & Frequencies (MHz) & TX Power Max & Duty Cycle (\%) & Emission BW & $\begin{array}{c}\text { Emission } \\
\text { (dBc) }\end{array}$ \\
\hline DME $^{2}$ & $1025-1150+/-100 \mathrm{kHz}$ & $40 \mathrm{dBm}$ & 0.03 & $0.5 \mathrm{MHz}$ & 50 \\
\hline $\mathrm{SSR}^{2,6}$ & $1090 \mathrm{MHz}+/-1 \mathrm{MHz}$ (Mode S) & see Table 3a & 0.03 (Mode S) & $2.6 \mathrm{MHz}(\mathrm{Mode}$ S) & 76 \\
\hline UAT $^{4}$ & 978 & see Table 3b & 0.0265 or 0.0403 & $0.9 \mathrm{MHz}$ & 70 \\
\hline ADS-B (ES) & $1090+/-1 \mathrm{MHz}$ & see Table 3a & $5 / 0.07$ & $2.6 \mathrm{MHz}$ & 76 \\
\hline
\end{tabular}

Table 2 - Spectral Mask Specifications for L-Band Civil Navigation Systems

\begin{tabular}{|c|c|c|c|c|c|c|c|c|}
\cline { 2 - 9 } \multicolumn{1}{c|}{} & \multicolumn{7}{c|}{ Spectral Mask Cha racteristics } \\
\hline System & $\begin{array}{c}\text { Ch+/- } \\
\text { (MHz) }\end{array}$ & $\begin{array}{c}\text { ATTEN } \\
(\mathrm{dB})\end{array}$ & $\begin{array}{c}\text { Ch+/- } \\
\text { (MHz) }\end{array}$ & $\begin{array}{c}\text { ATTEN } \\
(\mathrm{dB})\end{array}$ & $\begin{array}{c}\text { Ch +/ } \\
(\mathrm{MHz})\end{array}$ & $\begin{array}{c}\text { ATTEN } \\
\text { (dB) }\end{array}$ & $\begin{array}{c}\text { Ch +/- } \\
\text { (MHz) }\end{array}$ & $\begin{array}{c}\text { ATTEN } \\
\text { (dB) }\end{array}$ \\
\hline DME $^{2}$ & 0.8 & 23 & 2 & 38 & & & & \\
\hline SSR $^{2}$ & 1.3 & 3 & 7 & 20 & 23 & 40 & 78 & 60 \\
\hline UAT $^{4}$ & 1 & 18 & 2.25 & 50 & 3.25 & 60 & & \\
\hline ADS-B (ES) $^{5}$ & 1.3 & 3 & 7 & 20 & 23 & 40 & 78 & 60 \\
\hline
\end{tabular}

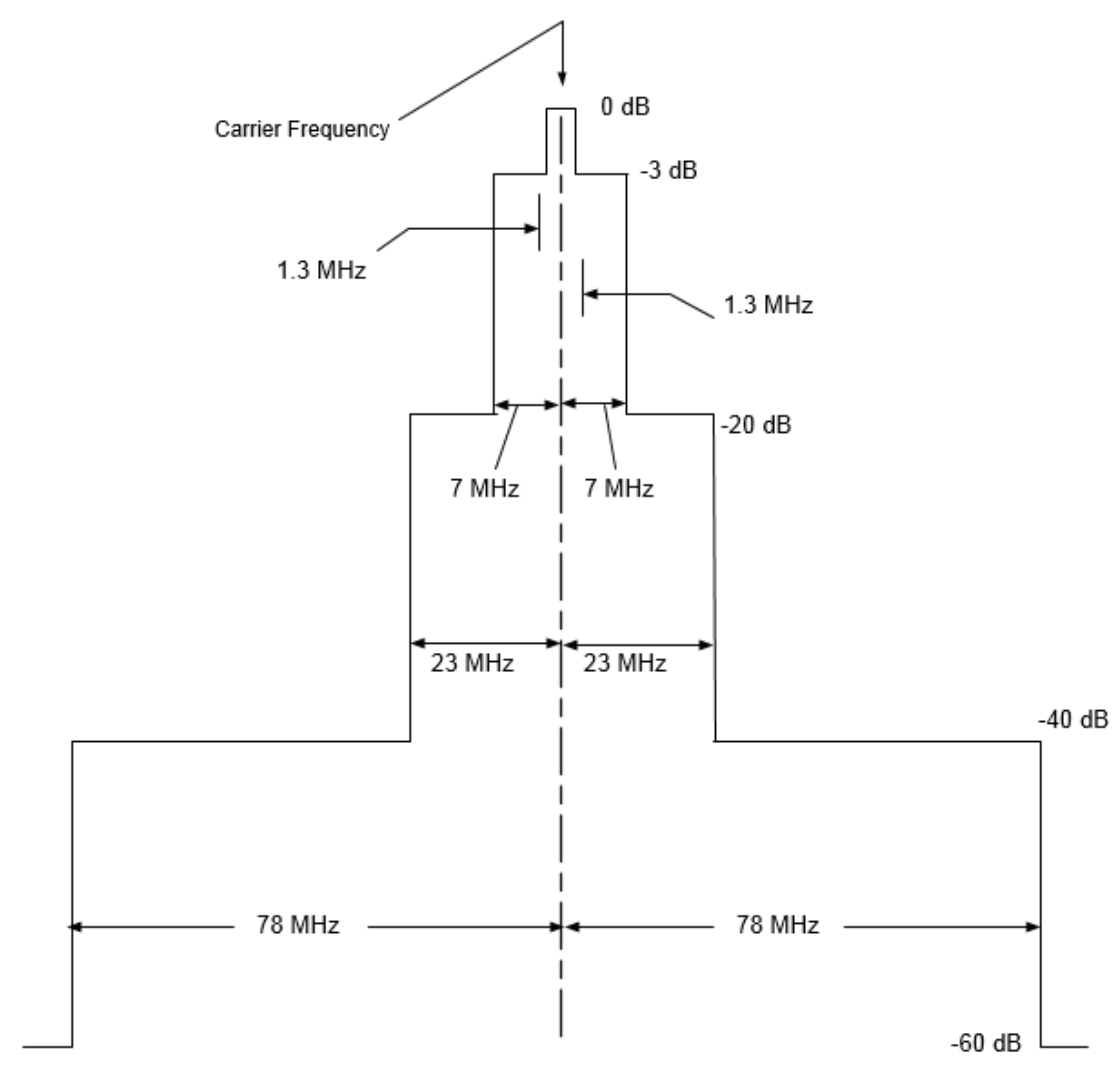

Figure 1 - Spectrum Limit Specification for Mode S Transponder Transmitter 


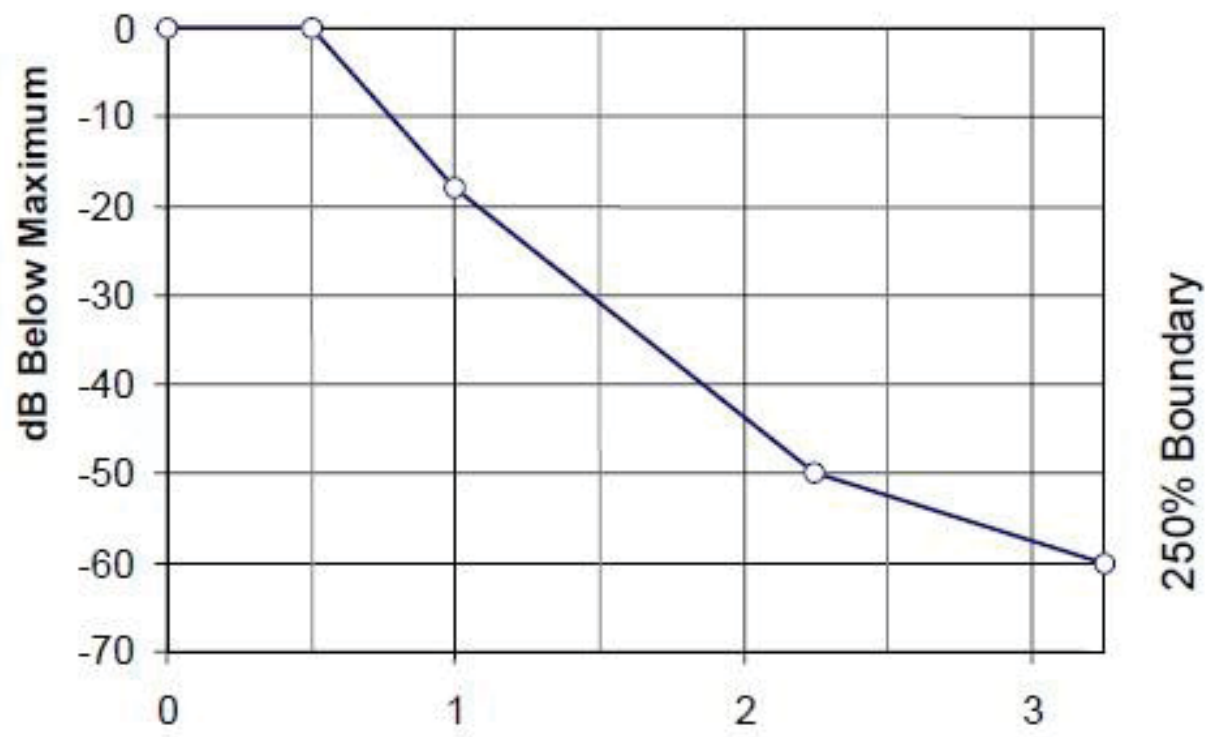

Frequency offset (MHz)

Figure 2 - Spectrum Mask for UAT ADS-B Transmitter

The key technical parameters of the airborne transmitters for these systems are shown in tables 1 and $2[2,4,5,6]$. Table 1 indicates the key transmitter parameters including operating frequencies, bandwidths, and transmit powers. Table 2 provides details of transmit spectral masks. Figures 1 and 2 show the specified transmit spectral masks for the 1090 Mode S transmitter and the UAT ADS-B transmitter, respectively.

RTCA Document DO-181D specifies the maximum peak RF output power for Mode 1090 ADS-B at the antenna input to be $57 \mathrm{dBm}(500 \mathrm{~W})$ [7]. During the inactive state between transmit pulses, the output power is specified to be $-50 \mathrm{dBm}$. For UAT ADS-B, RTCA document DO-282B specifies the maximum peak output power at the antenna input to be 54 $\mathrm{dBm}(250 \mathrm{~W})$ and during the inactive state between pulses the output power is specified to be $-80 \mathrm{dBm}$ [4]. This information provides the basis for analyses of the expected amount of power that may be input to a CNPC receiver in the 960-977 MHz band of interest.

\section{CNPC TEST RESULTS}

NASA and Rockwell Collins have been conducting flight tests of prototype UAS CNPC radios operating in both LBand and C-Band. In more recent flight tests, radiofrequency interference has been observed that was not seen in previous flight tests. The appearance of this interference corresponds to installation of new ADS-B units, a Universal Access Transceiver (UAT) and a 1090 ES ADS-B transponder. However there are a number of significant differences between the several sets of flight tests such that it is not certain that at least some of the interference seen in the recent flight tests was not also present but not visible in earlier tests.

It is recognized that the level of electromagnetic coupling between the airborne CNPC and navigations systems may be specific to the NASA S-3 research aircraft and its antenna placements. Other aircraft may have similar or other installation-specific issues due to aircraft size, materials, and/or navigation electronics packages.

Although more extensive investigations are planned, results of flight testing and laboratory measurements presented below indicate that the 1090 ES ADS-B transponder may be responsible for at least some of the observed interference. Test results and interpretations of these results are presented below.

\section{Interference Observations from CNPC Flight Tests}

Data collected from the April 11, 2014 flight test in Ohio, USA is presented in Figure 3. Four individual signal strength traces are plotted in the top section of the figure; two traces for uplink signals transmitted from the ground station to the NASA S-3 flight test aircraft, and two similar signals transmitted from the S-3 and received at the ground station. The next four sections in figure 3 show corresponding data packet loss for four types of uplink and downlink transmitted signals (designated as UL1, UL20, DLC2, and DL video signals with corresponding bandwidths of $75 \mathrm{kHz}, 875 \mathrm{kHz}$, $75 \mathrm{kHz}$, and $650 \mathrm{kHz}$ respectively). The bottom three sections 
in figure 3 show the range (distance between the aircraft and the ground station), aircraft altitude, and aircraft roll (indicating flight maneuvers). The data in all of the traces are precisely aligned in time, shown on the $\mathrm{x}$-axis. The CNPC waveform utilizes time-division duplexing (TDD) between the uplink and downlink, with each signal allocated a subsection of each $50 \mathrm{~ms}$ TDD frame. Using this technique, the uplink and downlink are non-interfering and can both be tested at 20 times per second.

When the aircraft is within close range of the ground station, the received signal strengths are relatively high and all four traces track one another closely. However, as separation distances increase and received signal power drops below approximately $-100 \mathrm{dBm}$, the airborne radio records multiple closely-spaced "spikes" in the UL1 and UL20 uplink data traces (blue and light blue colors). These are most visible in the UL1 trace during the period of 17:03 through 17:29. While the S-3 aircraft was flying outbound at a range of approximately $70 \mathrm{nmi}$, the signal strength received at the aircraft radio was approaching its sensitivity limit near -119 $\mathrm{dBm}$. While the underlying signal strength (red) trace trends further downward, the amplitudes of the spikes (blue) appear to be staying relatively constant. The April 11, 2014 flight is the first test where this effect has been recorded; previous tests have not displayed this effect. The spikes are not visible in the DLC2 or DL video "downlink" traces recorded by ground station radio. As noted above, subsequent investigation revealed that the ADS-B transponder and other equipment on the S-3 aircraft had been changed prior to the April test series. The new ADS-B unit, equipped with an extended squitter transmitter at $1090 \mathrm{MHz}$, was suspected to be a probable cause of at least some of the observed spikes. Although the transponder is designed to operate outside of the 960-977 MHz CNPC radio band, it is apparent that the transponder transmissions are being received by the CNPC radio on the aircraft. Figure 5 shows the relative locations of the UAS CNPC L-Band antenna and the 1090 ES ADS-B antenna on the S3 aircraft. In-flight tests were typically performed with the aircraft operating at constant altitude, attitude, and airspeed. Airframe shadowing can be observed during course reversals.

To investigate the possible source of the spikes, a test was conducted April 23, 2014 during flight tests in Ohio, USA in which the ADS-B transponder would be briefly turned off during flight. Operating conditions were identical to those of the April 11, 2014 test, including CNPC radio configuration, inbound/outbound flight path, and downrange distance. For this test, the flight altitude was increased to eliminate the possibility of line-of-sight interruption by terrain obstruction.
In coordination with local air traffic controllers, the transponder was de-powered for approximately five minutes during the outbound pass, then reactivated for reversal and the inbound pass. Data for the test is presented in figure 4.

The airborne ADS-B transponder was in operation from the time of take-off until the S-3 was approximately $80 \mathrm{nmi}$ downrange from the GRC ground station. At this time the received signal strength at the aircraft was approximately $103 \mathrm{dBm}$ which is above the sensitivity limit of the radio and at a level that the spikes would become visible in the data trace. From 14:48 the 14:53 the transponder output was deactivated while the aircraft continued on a straight and level flight path, extending to approximately $110 \mathrm{nmi}$ downrange. The data trace in figure 4 clearly shows that some spikes have abated throughout the five-minute period, especially when compared to the same airspace and flight conditions on the inbound run (15:08 through 15:13) when the ADS-B transponder was back in operation. The ADS-B deactivation appears to have greater impact on the UL1 trace, as it shows greater reduction in number of spikes. The widerband UL20 signal does not experience the same cleansing during the ADS-B off period, indicating that an interferer is still present. Noise spikes were not visible in either of the downlink signals received by the ground station. The overlap of the uplink and downlink data traces indicates the strong similarity of the characteristics of the channels, and also gives a forecast of how the uplink signal should be received once the interfering signal is removed.

The outbound portion of the flight test, which began at 14:33 UTC, yielded high quality data transmission (0-percent losses) both ground-to-aircraft and aircraft-to-ground until a few spikes were recorded at the aircraft beginning at approximately 14:44. The ADS-B transponder was deactivated at 14:48 which caused the spikes in the UL1 trace to disappear. The ADS-B transponder was re-activated at 14:53, at which time the UL1 spikes returned. At 14:53, the UL1 frame loss trace shows signal deterioration prior to the onset of $\mathrm{C} 2$ frame loss. This indicates that the interfering signal is having negative impact on the uplink CNPC channel.

On the inbound portion of the flight, the impact of the interfering signal is visible in the UL1 frame loss trace from 15:08 through 15:13. The multitude of vertical lines indicate that the radio is experiencing frame losses. Over the same time period, the C2 frame losses are much lower, resulting little visible trace on the grid. 


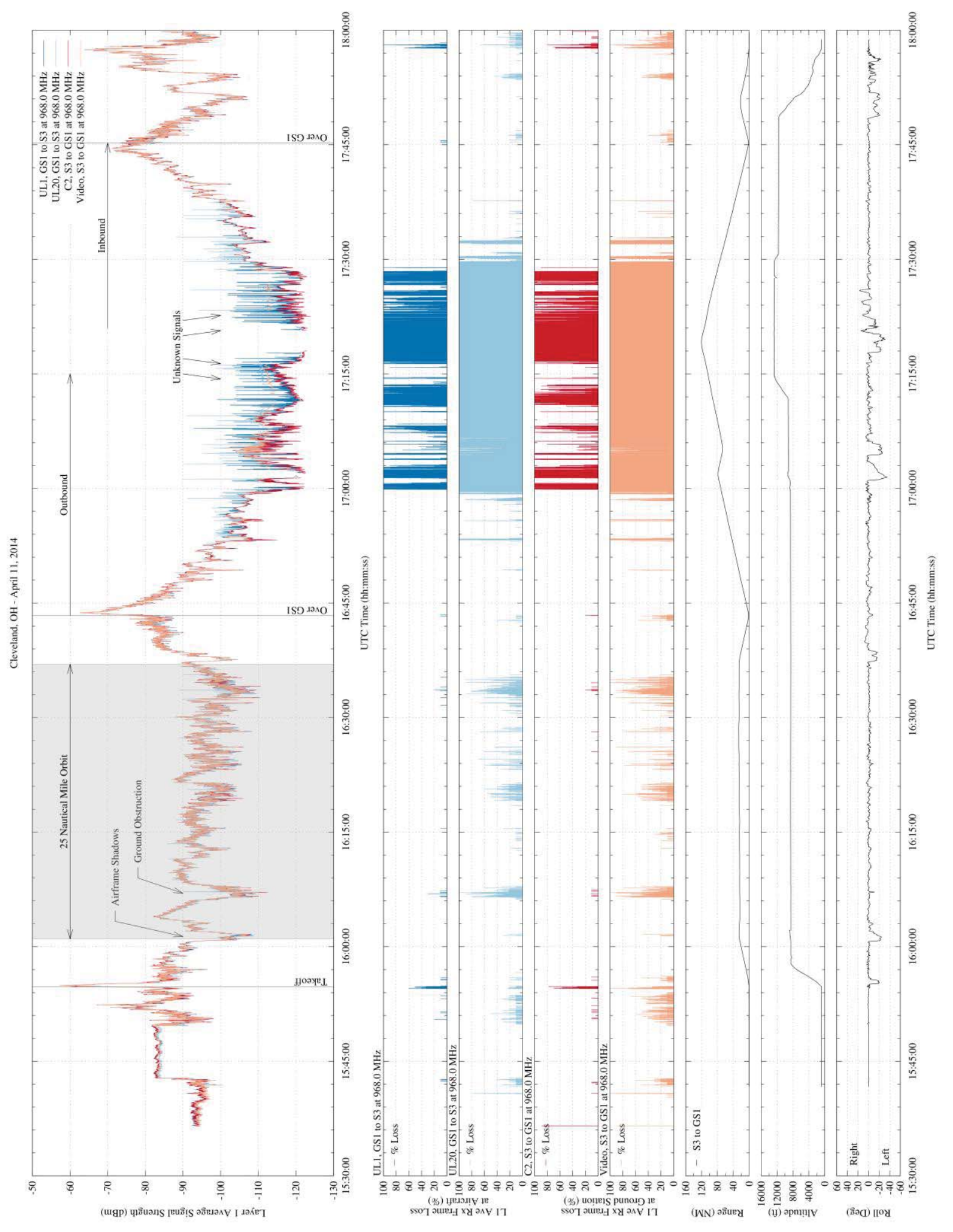

Figure 3 - Observation of unknown interference signals during the flight test of 11 April 2014. 


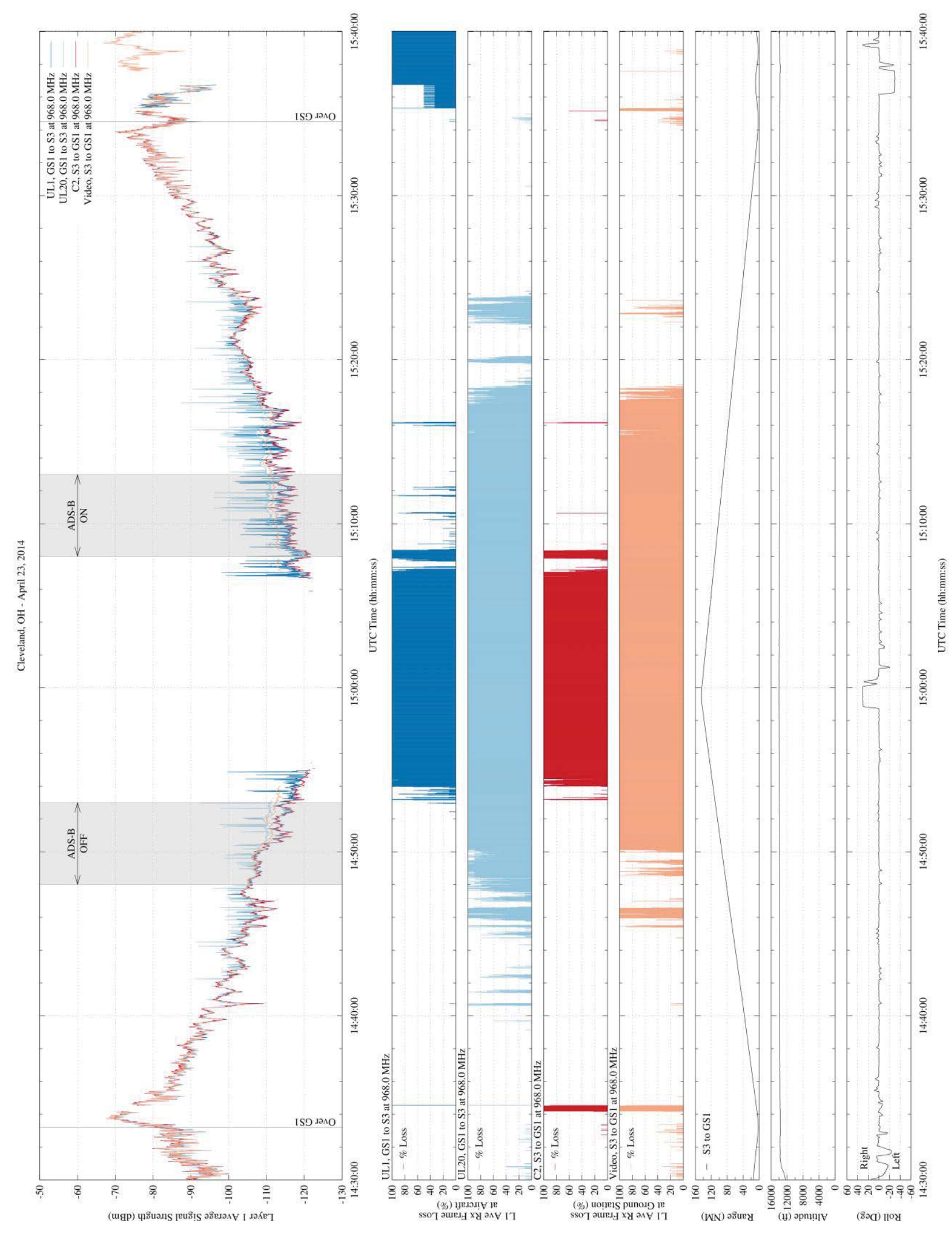

Figure 4 - ADS-B transmitter on-off test during the flight test of 23 April 2014. 


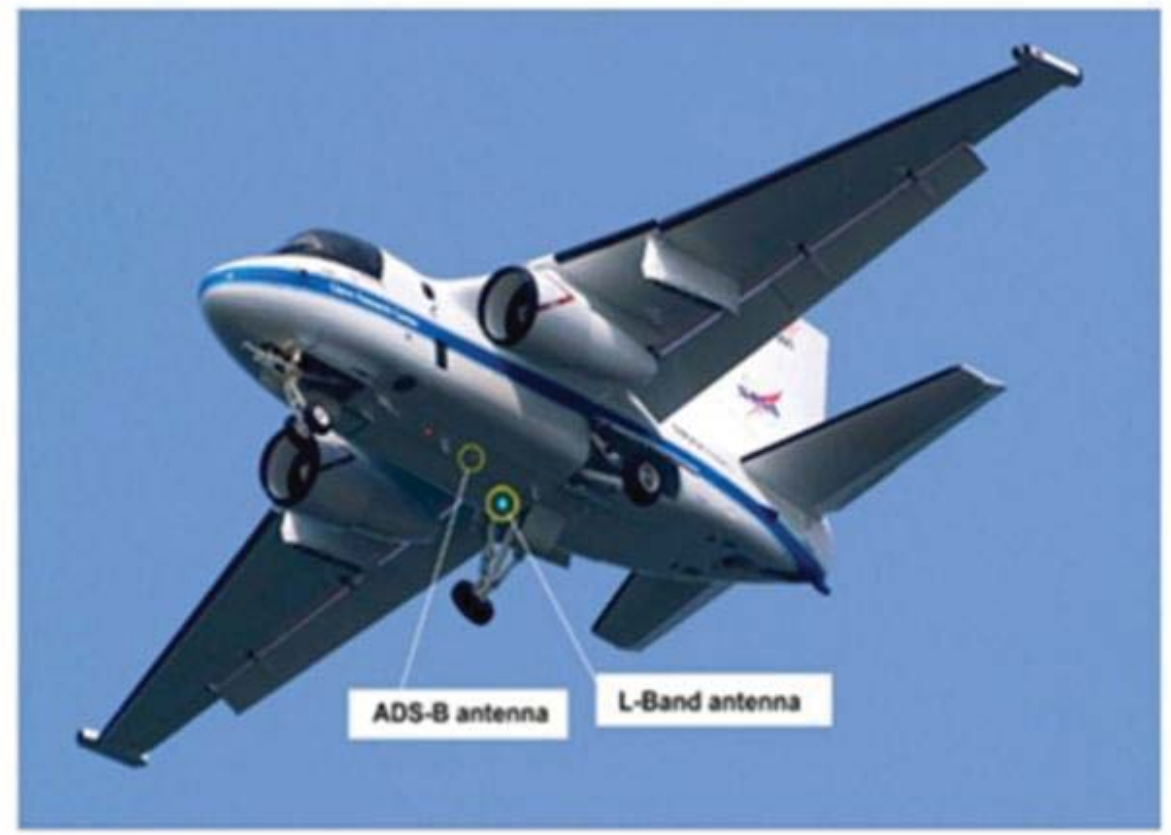

Figure 5 - Locations of L-Band CNPC and 1090ES antennas on the NASA S3 aircraft.

\section{Laboratory Tests}

Initial investigations were performed in the NASA UAS laboratory to examine the possible source(s) of the unwanted signals in the 960-977 MHz CNPC band. Measurements were made by connecting a spectrum analyzer directly to the antenna port of the UAT and 1090 ES transmitters. A largevalue attenuator was placed at the output of the transmitter to protect the input of the spectrum analyzer. These measurements were made as part of a cursory interference investigation and do not include detailed calibrations, hence this data is presented for qualitative observation only. The UAT transponder transmits at a center frequency of $978 \mathrm{MHz}$ and the $1090 \mathrm{ES}$ transponder transmits at $1090 \mathrm{MHz}$.

Figure 6 shows the output of the UAT transmitter at $978 \mathrm{MHz}$ and $968 \mathrm{MHz}$. The uncalibrated difference is approximately $75 \mathrm{~dB}$. Figure 7 shows the output of the $1090 \mathrm{ES}$ transmitter at $1090 \mathrm{MHz}$ and $968 \mathrm{MHz}$. The uncalibrated difference, considering the additional $10 \mathrm{~dB}$ of attenuation added for the measurement at $1090 \mathrm{MHz}$, is approximately $45 \mathrm{~dB}$.

These measurements indicate that a substantial amount of output power is being transmitted within the $960-977 \mathrm{MHz}$ band by both the UAT and 1090 ES units. Further investigation is needed to determine the level of signal coupling between the ADS-B transmitters and CNPC receiver for the NASA S3 configuration.

\section{Interpretation of Results}

The flight tests of April 2014 indicated that interference likely resulting from co-site interference on the S3 aircraft was present. The interference can be clearly seen when the aircraft is sufficiently far from the ground station such that CNPC received signal strength is below $-90 \mathrm{dBm}$ as shown in Figures 3 and 5.

The flight test in which the $1090 \mathrm{ES}$ transmitter is turned off for a portion of the test indicates that a substantial portion of the interference is due to the out-of-band emissions from the 1090 ES as shown in Figure 5. The source of the other interference observed when the 1090 ES is turned off has not yet been determined.

Laboratory tests of the UAT and 1090 ES ADS-B transponders (Figures 6 and 7) indicate a substantial amount of out-of-band power emitting from these units. Although coupling between the ADS-B and CNPC antennas has not been characterized, the out-of-band power levels measured in the NASA UAS laboratory appear to be sufficient to create data loss in the CNPC receiver.

The ADS-B interference issue described in this section and other co-site effects will be examined in future NASA test activities. Factors including antenna placement, RF filtering, and radio waveform configuration/synchronization will be investigated in subsequent tests. 

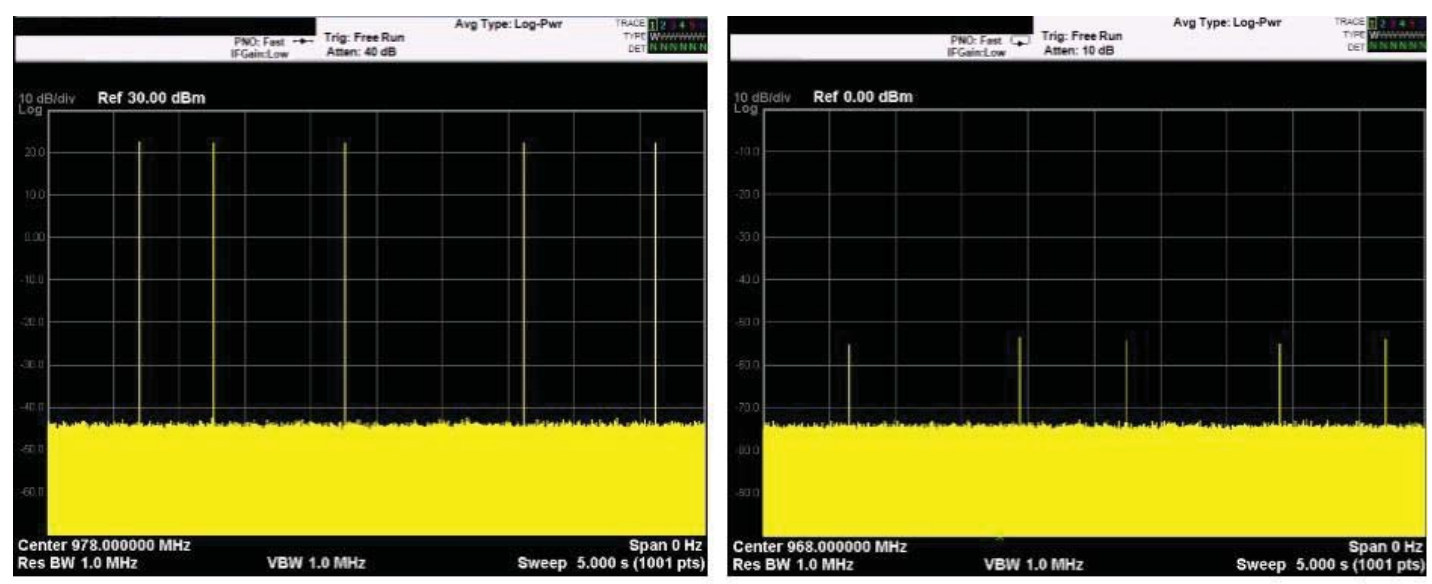

Figure 6-UAT transmitter output at $978 \mathrm{MHz}$ (left) and $968 \mathrm{MHz}$ (right)
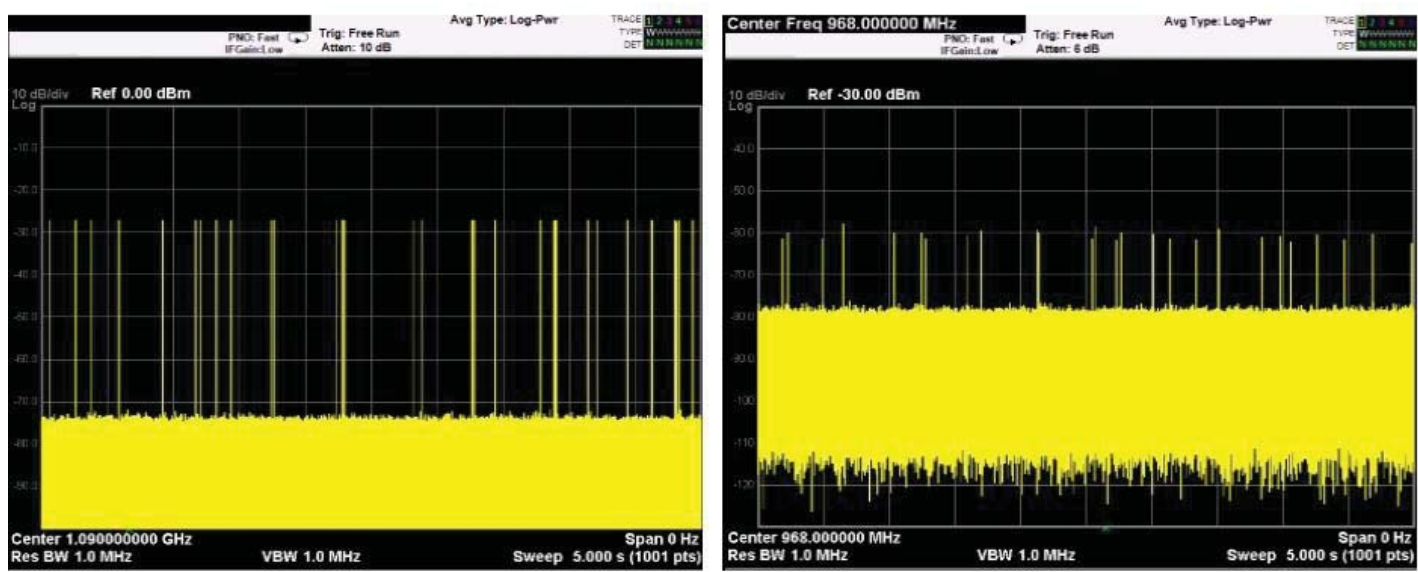

Figure 7 - $1090 \mathrm{ES}$ transmitter output at $1090 \mathrm{MHz}$ (left) and $968 \mathrm{MHz}$ (right). Note an additional $10 \mathrm{~dB}$ attenuator was added at $1090 \mathrm{MHz}$ (left figure).

\section{IMPACT ON CNPC SPECTRUM}

The spectrum requirements for UAS CNPC have been defined by the ITU-R for the purpose of determining spectrum allocations, using projections of the expected UA density for the year 2030 [8].

The data transfer requirements for a single UA support several functions: command and control; air traffic control (ATC) relay; sense and avoid (S\&A); and video and weather radar. Command and control includes command and navigational signals sent to the UA from the UA control station (UACS) and position, telemetry and navigational information sent from the UA to the UACS. Air traffic control relay consists of voice and data commands from ATC to the UACS through UA. The data requirements for these functions were considered for six phases of flight: taxiing; takeoff; initial climb; cruise (en-route); approach; and landing.

The data requirements thus derived were aggregated over the projected 2030 UA densities to arrive at spectrum requirements. For both UA density methodologies, the terrestrial LOS spectrum requirement was determined to be $34 \mathrm{MHz}$. For the satellite BLOS component, the spectrum requirement is between $46 \mathrm{MHz}$ and $56 \mathrm{MHz}$, dependent upon the type of satellite system used (spot beam or regional beam). Thus the maximum spectrum requirements for UAS are:

$34 \mathrm{MHz}$ for LOS terrestrial systems.

$56 \mathrm{MHz}$ for BLOS satellite systems. 
The 5030-5091 MHz part of C-band was allocated to the aeronautical mobile (route) service $(A M(R) S)$ at the WRC12. This allocation provides for a CNPC LOS system. However, this band also contains an aeronautical mobile satellite (route) service (AMS(R)S) allocation. This allocation can support CNPC BLOS. Finally, an AM(R)S allocation was made to the L-Band (960-1164 MHz) at WRC07. As mentioned, the $960-977 \mathrm{MHz}$ portion of L-Band is considered suitable for LOS CNPC.

Thus there are currently three bands that could provide both LOS and BLOS CNPC for UAS. The total bandwidth of 78 $\mathrm{MHz}$ from these three bands does not fulfill the combined 90 $\mathrm{MHz}$ requirement for LOS and BLOS CNPC. Since the LOS component will require a substantial portion of the C-Band allocation, BLOS CNPC requires some further allocation. This subject is being considered by WRC-15 under its Agenda Item 1.5.

Co-site interference in L-Band will impact the CNPC spectrum situation. Although $17 \mathrm{MHz}$ is under consideration (960-977 MHz), co-site interference will reduce the effective use of this band. At a minimum, UA operating in nonsegregated airspace will need to meet the same requirements for manned aircraft in terms of ADS-B. In Table 2, the \pm 1 $\mathrm{MHz}$ mask for UAT requires only $18 \mathrm{~dB}$ emission attenuation; the $\pm 2.25 \mathrm{MHz}$ mask requires $50 \mathrm{~dB}$ emission attenuation. The UAT nominal transmit power of $40 \mathrm{~W}$ suggests that the CNPC receiver for UA equipped with UAT ADS-B systems might be restricted to an operational bandwidth of $960-975 \mathrm{MHz}$ to avoid severe co-site interference. Although the 1090 ES ADS-B transmit frequency is farther removed from that $960-977 \mathrm{MHz}$ band than UAT, the transmit power is higher. As the NASA flight tests have shown, at link distances within the expected range of CNPC LOS systems, 1090 ES is capable of creating cosite interference.

Considering the maximum transmit powers possible for 1090ES (57 dBm) and UAT (54 dBm), systems that operate well below the specified transmit masks shown in figures 1 and 2 can still produce output powers in the $960-977 \mathrm{MHz}$ band of up to $-3 \mathrm{dBm}$ and $-6 \mathrm{dBm}$, respectively. The amount of this energy that is coupled into the CNPC antenna and is thus present at the CNPC receiver input is highly dependent on physical installation and antenna separation. Since many UA are relatively small compared to typical manned commercial aircraft, opportunities for mitigating co-site interference through optimized installation and maximized antenna separation are limited. Intersystem isolation in excess of $100 \mathrm{~dB}$ might be required, which may be very difficult to achieve.

Co-site interference will have the effect of reducing the range of flight obtainable, since the CNPC radio will only be able to function at distances from the ground station that enable the CNPC receiver to overcome the constant level of interference produced by other on-board equipment. Closer spacing of ground stations would then be required. With these ground stations still operating at the same transmit power, frequency re-use factors would be reduced, leading to a more rapid depletion of available spectrum.

Some mitigation of the interference effects will be needed, for example blanking the receiver during 1090 ES transmission pulses. Mitigation will have the effect of reducing CNPC throughput, requiring additional or wider CNPC channels to meet data transfer requirements. Another possible mitigation is to incorporate additional filtering at the 1090 ES output. The potential impact of this additional filtering requires investigation. Overall the effective use of the $960-977 \mathrm{MHz}$ band is potentially reduced regardless of which ADS-B system is installed.

With potentially several $\mathrm{MHz}$ of the 960-977 $\mathrm{MHz}$ band not effectively available for LOS CNPC, and the possible additional impact of reduced range of CNPC operation, more of the C-Band allocation will be required. Instead of $17 \mathrm{MHz}$ of L-Band and $17 \mathrm{MHz}$ of C-Band for LOS CNPC, perhaps 20 or more $\mathrm{MHz}$ of $\mathrm{C}$-Band will be required. This in turn reduces the amount of $\mathrm{C}$-Band spectrum available to BLOS CNPC, with a potential impact on the financial viability of a future C-Band satellite deployment to provide BLOS CNPC.

\section{NEXT STEPS}

The ADS-B interference issue described in this paper and other co-site effects are planned to be examined in future NASA test activities as time and resources permit.

Further understanding of the co-site interference, its impact on CNPC radio performance and spectrum requirements, and development and implementation of mitigation methods will involve both theoretical and empirical data analysis. Factors including antenna placement, RF filtering, and radio waveform configuration/synchronization are under consideration for investigation in subsequent tests. Equipment expressly configured for this purpose will need to be automated and mounted in a controlled area in the aircraft. Comprehensive test plans are under development, including electromagnetic interference (EMI)/electromagnetic compatibility (EMC) testing [9] insuring that the on board ADS-B equipment does not provide an interference source for UAS CNPC or other installed systems on the aircraft. Laboratory testing using L-Band navigation and prototype CNPC is also under consideration within the RTCA SC-228 but no plans have been finalized. Results from such tests may support additional requirements for navigation equipment installed on UAS aircraft with L-Band CNPC radios.

Further analysis is planned by NASA using the Visualyse Professional software [10] to analyze interference into proposed CNPC links for UAS in the 960 to $977 \mathrm{MHz}$ band. Several in-band and adjacent-band systems which may cause interference will be modeled. Particular attention will be addressed to the following: 
The UAT ADS-B system, operates on a single frequency near the edge of the CNPC allocation at $978 \mathrm{MHz}$ with a maximum transmission power of $54 \mathrm{dBm}$.

1090 Extended Squitter (1090 ES) also and ADS-B system, operates at $1090 \mathrm{MHz}$ with a transmitter power of $57 \mathrm{dBm}$.

Secondary surveillance radar (SSR) operates at $1090 \mathrm{MHz}$ with a maximum transmission power of $57 \mathrm{dBm}$.

Although they are military-use systems, because the Joint Tactical Information Distribution System (JTIDS) and the Multifunctional Information Distribution System (MIDS) have an overlapping sub-band over 969-1008 $\mathrm{MHz}$ with a maximum transmission power of $60 \mathrm{dBm}$, they will also be investigated.

\section{SUMMARY}

The use of the L-Band frequency range 960-977 $\mathrm{MHz}$ to support will provide needed spectrum for UAS CNPC systems, in terms of both bandwidth and availability requirements. However, the presence of several other aeronautical navigations systems requiring on-board transmitters in nearby portions of L-Band leads to concerns about co-site interference into the CNPC on-board receiver. RTCA SC 203 and SC-228 have noted the potential co-site interference problem and recommended further testing and analysis to characterize its extent. NASA flight tests of a prototype CNPC receiver has shown that interference apparently due to co-site issues has been observed.

Co-site interference in L-Band will have the potential impact of reducing the effective amount of L-Band bandwidth available for CNPC LOS operations. As a result, more CBand bandwidth will be required, affecting the use of that band for BLOS systems.

RTCA has recognized that further testing and analysis is needed to more fully understand the co-site interference issue and develop methods to deal with it. NASA and other RTCA members are considering ways to meet this requirement within existing resources. NASA plans to conduct additional investigations through flight testing when there are opportunities within the planned flight test campaign as time and resources permit, and through lab testing. NASA also plans to conduct analysis through modeling and simulation tools. The availability of a UAS CNPC prototype radio and validated models of the radio's performance make it possible to conduct these investigations.

\section{REFERENCES}

[1] Box, F., Globus, L., D, Wilson, W. J., and Ashley, J., "Compatibility of Terrestrial L-Band CNPC with In-Band and Adjacent-Band Systems", RTCA SC-203-CC021, March 2013.

[2] "B-AMC Interference Analysis and Spectrum Requirements", EUROCONTROL Report CIEA15_EN503.1122 October 2007.

[3] Micallef, J., Womersley, R., Dunkley, S. and Casewell, I., "L-Band Interference Scenario Characterisation”, EUROCONTROL Report P1031D005, 25 August 2009.

[4] RTCA DO-282-B "Minimum Operational Performance Standards for Universal Access Transceiver (UAT) Automatic Dependent Surveillance - Broadcast (ADS-B)", 2 October 2009.

[5] RTCA DO-260-B "Minimum Operational Performance Standards for 1090 Extended Squitter Automatic Dependent Surveillance - Broadcast (ADS-B) and Traffic Information Services - Broadcast (TIS-B), 2 December 2009.

[6] ITU-R Report M.2171 "Characteristics of unmanned aircraft systems and spectrum requirements to support their safe operation in non-segregated airspace", December 2009.

[7] RTCA DO-181-E "Minimum Operational Performance Standards for Air Traffic Control Radar Beacon System/Mode Select (ATCRBS/Modes S) Airborne Equipment”, 17 March 2011.

[8] ITU-R Report M.2205 "Results of studies of the AM(R)S allocation in the band 960-1 $164 \mathrm{MHz}$ and of the AMS(R)S allocation in the band 5 030-5 $091 \mathrm{MHz}$ to support control and non-payload communications links for unmanned aircraft systems", November 2010.

[9] Federal Aviation Administration Advisory Circular AC No. 20-265 "Airworthiness Approval of Automatic Dependent Surveillance - Broadcast (ADS-B) Out Systems", 21 May 2010.

[10] www.transfinite.com 


\section{BIOGRAPHIES}

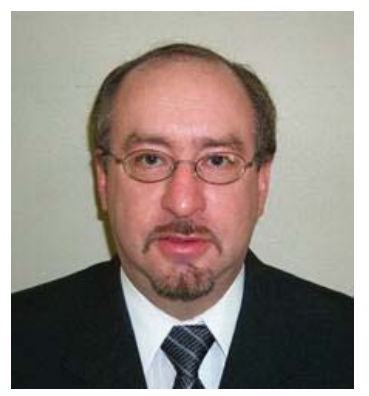

Robert J. Kerczewski has been involved with research and development of satellite and aeronautical communications systems and applications for the Analex Corporation (1982-1986) and NASA (1986-present). He holds a BEE degree from Cleveland State University (1982) and an MSEE degree from Case Western Reserve University (1987). He is currently the Spectrum Element Manager for the NASA's Unmanned Aircraft Systems Integration in the National Airspace System (UAS in the NAS) Communications Sub Project.

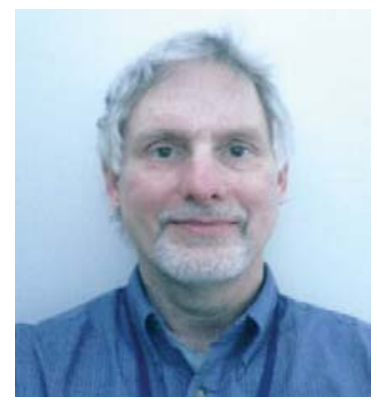

William D. Bishop is currently involved with the research, design and development of Unmanned Aircraft Systems (UAS) Control Non-Payload Communications (CNPC) Systems at NASA in the Datalink and Spectrum Sub-Project areas (2010-present). He also has extensive, comprehensive wireless industry experience (commencing in 1986) in the $R F$ design, optimization and management of wireless networks supporting all U.S. carriers in all currently deployed voice and data technologies. His early efforts include the design, development and practical use of the Dual-Cell Concept (FOA - GTE Mobilnet, 1987), which increased offered load capacity in congested areas within a traditional reuse plan. He holds a BEE degree from Cleveland State University Fenn College of Engineering (1998), as well as an FCC General Radiotelephone License (1986).

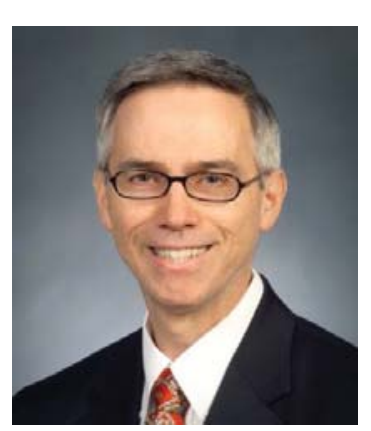

Jeffrey $D$. Wilson received the B.S. degree in physics magna cum laude from Bowling Green State University in 1976, and the M.S. and Ph.D. degrees in physics from the University of Illinois at Urbana-Champaign in 1978 and 1983, respectively. Since 1983, Dr. Wilson has been associated with the vacuum electronics microwave amplifier research group at NASA Glenn Research Center, Cleveland, Ohio. He spent the 1984-1985 academic year in postdoctoral study with the Air Force Thermionic Electronics Research (AFTER) Program at the University of Utah. His research efforts have focused on computational techniques to enhance the power, efficiency, and performance of coupled-cavity, helical, and terahertz wave traveling-wave tubes (TWT's), the electromagnetic properties of metamaterials, and interference issues in RF communications systems. Dr. Wilson is a Senior Member of IEEE.

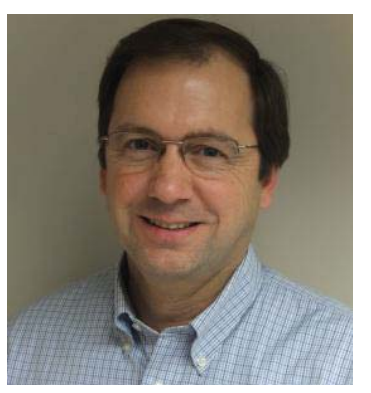

Kurt Shalkhauser received the B.S.E.E. degree from the Pennsylvania State University in 1983 and M.S.E.E. degree from University of Toledo in 1988. Kurt began his career at NASA in 1982 working in space communications research and development. More recent work has included characterization and development of a reverberant EMI test chamber for full scale spacecraft, and thermal-vacuum testing of communications payloads, rocket propulsion systems, and atmospheric research equipment. Kurt is currently Datalink Element Manager for NASA's Unmanned Aircraft Systems integration in the National Airspace System (UAS in the NAS) communications subproject. 